\section{Three-Color Imaging Using Fluorescent Proteins in Living Zebrafish Embryos}

BioTechniques 31:66-72 (July 2001)

\begin{abstract}
The zebrafish embryo is especially valuable for cell biological studies because of its optical clarity. In this system, use of an in vivo fluorescent reporter has been limited to green fluorescent protein (GFP). We have examined other fluorescent proteins alone or in conjunction with GFP to investigate their efficacy as markers for multi-labeling purposes in live zebrafish. By injecting plasmid DNA containing fluorescent protein expression cassettes, we generated single-, double-, or triple-labeled embryos using GFP, blue fluorescent protein (BFP, a color-shifted GFP), and red fluorescent protein (DsRed, a wild-type protein structurally related to GFP). Fluorescent imaging demonstrates that GFP and DsRed are highly stable proteins, exhibiting no detectable photoinstability, and a high signalto-noise ratio. BFP demonstrated detectable photoinstability and a lower signal-to-noise ratio than either GFP or DsRed. Using appropriate filter sets, these fluorescent proteins can be independently detected even when simultaneously expressed in the same cells. Multiple labels in individual zebrafish cells open the door to a number of biological avenues of investigation, including multiple, independent tags of transgenic fish lines, lineage studies of wild-type proteins expressed using polycistronic messages, and the detection of protein-protein interactions at the subcellular level using fluorescent protein fusions.
\end{abstract}

\section{INTRODUCTION}

Researchers have capitalized on the optical clarity of the zebrafish embryo to demonstrate the utility of analysis of living embryos through lineage studies with the green fluorescent protein (GFP) from the bioluminescent jellyfish Aequorea victoria (3). This method has greatly simplified transgene detection, promoter discovery, and cell lineage analysis by providing a visual marker in a live organism $(8,9)$. The potential of fluorescent protein use in zebrafish is only beginning to be explored.

In addition to the targeting of genes and regulatory sequences, fluorescent proteins have opened the door for lineage and cell biology in living eukaryotic cells $(4,11)$, but the use of multiple markers in vivo has been lacking in zebrafish. Spectrally shifted mutant versions of GFP (blue, cyan, yellow, and red) have become available (5). Recently, a red fluorescent protein (DsRed) was cloned from non-bioluminescent Discosoma species based on suspected conservation between GFP-like proteins (10). We demonstrate that under the control of the Xenopus EF1 $\alpha$ promoter, green, blue, and red fluorescent proteins (GFP, BFP, and DsRed, respectively) are useful labels in the fish individually, in pairs, or in triplicate. We show that the onset of expression occurs early in development and that GFP and DsRed are exceptionally photostable, while $\mathrm{BFP}$ is susceptible to photolysis. We have developed fluorescent filter sets that allow each protein to be readily detected without signal overlap or false color enhancement. We anticipate the use of multiple fluorescent proteins to facilitate insertional mutagenesis, enhancer and gene trapping techniques, and protein-protein interaction investigations using fluorescently labeled protein constructs in single cells.

\section{MATERIALS AND METHODS}

\section{Fish Maintenance, Injections, and Photography}

Wild-type zebrafish were purchased from Segrest Farms (Gibstonton, FL, USA). Adult fish maintenance and embryo collections followed standard protocols (13). One-cell embryos were injected with $50 \mathrm{pg}$ plasmid DNA as described (6). Embryos were raised at $31^{\circ} \mathrm{C}$ and photographed on a Zeiss Axioplan2 $2^{\mathrm{TM}}$ microscope (Carl Zeiss, Thornwood, NY, USA) with a model i308 Integrating Low Light Camera (MicroImage Video Systems, Boyertown, PA, USA). Images were captured with Adobe ${ }^{\circledR}$ Premiere ${ }^{\circledR}$ version 4.2 software and rendered in Adobe Photoshop $^{\circledR}$ version 5.0.

\section{Injection Constructs}

pT- $\beta$-GM2 was a generous gift of Deanna Mohn and Perry Hackett and includes the GFP/GM2 (9) open reading frame (ORF) under control of the Xenopus EF1 $\alpha$ promoter (7).

pXeX-BFP was generated by PCR to add $5^{\prime} S a l \mathrm{I}$ and $3^{\prime} \mathrm{ClaI}$ sites to the ORF of BFP in pFRED, a BFP variant derived from spectrally shifted GFP mutants (Reference 12 and G.N. Pavlakis, personal communication). The oligonucleotides used were 5': AD1 (5'-ACGGGTCGACAAGAAATGGCTAGCAAGGG-3') and 3': AD2 (5'-CCATCGATCCTTACTTGTACAGCTCGTCCATGC-3'). Digestion of the 740-bp PCR fragment with SalI and ClaI (New England Biolabs, Beverly, MA, USA) and subsequent ligation with T4 DNA ligase (Promega, Madison, WI, USA) into the 3137-bp SalI, ClaI fragment of pXeX-GFP (2) resulted in $\mathrm{pXeX}$-BFP.

pXeX-DsRed was cloned by adding the same 5'SalI and 3' ClaI sites to the ORF of pDsRed1-N1 (Clontech Laboratories, Palo Alto, CA, USA). The oligonucleotides used were DsRed1Sal15' (5'-TTAGTCGACCACCATGGTGCGCTCCT-3') and DsRed1ClaI3' (5'-GGCTTAATCGATGCTACAGGAACAGGTGGT-3'). The 698-bp PCR product was digested with SalI and ClaI and ligated into the 3137-bp SalI and ClaI-digested fragment of pXeXBFP, exchanging the BFP ORF for the DsRed ORF.

\section{Time Course Photostability Assay}

To assay susceptibility to photodegradation, a strongly fluorescing 24hpf embryo representing each fluorescent protein was mounted in methyl cellulose on a single glass slide as described (13). Embryos were illuminated for $15 \mathrm{~min}$ with light transmitted through the appropriate excitation filter for the corresponding fluorescent protein. Images were captured as described above, holding exposure times constant.

\section{Real-Color Imaging}

Capturing real color was possible with filter sets from Chroma Technology (Brattleboro, VT, USA). The Endow 
GFP Longpass Emission filter set (1pGFP) consisted of an HQ470/40x excitation filter, a Q495LP dichroic beam splitter (DCBS), and an HQ500LP emission filter. Endow GFP Bandpass Emission (bp-GFP) utilized the HQ470/40x, Q495LP DCBS, but an HQ525/50m emission filter. GFP images were captured with this filter set except in Figure 3, A-C, which used the lp-GFP filter set. The Blue GFP Bandpass Emission filter set (b-GFP) had a D390/22x excitation filter, a 420DCLP DCBS, and a D460/50m emission filter. The rhodamine filter set used D546/10x, an R+B Phycoerythrin excitation filter, a 560DCLP DCBS, and an E570LP emission filter from the FM143 filter set.

\section{RESULTS}

We were interested in determining the onset of expression of each fluores- cent protein to assess its reliability as a temporal marker. The expression vector $\mathrm{pXeX}(7)$ is effective for ectopic expression of GFP in zebrafish $(1,2)$. pXeX contains the Xenopus EF $1 \alpha$ enhancer/promoter and a simian virus 40 (SV40) polyadenylation signal (7). All three of our injection constructs were based on $\mathrm{pXeX}$. Similar to previous reports $(1,2,8)$, we first detected GFP from $\mathrm{pT}-\beta-\mathrm{GM} 2$ at the late-sphere stage (4 h of development; Figure 1, A and D), approximately $1 \mathrm{~h}$ after zygotic transcription is initiated in the zebrafish embryo. GFP is thus a very useful marker for the detection of early expressed zygotic genes. BFP expression from $\mathrm{pXeX-BFP}$ was detected later at the shield stage $(6 \mathrm{~h}$ of development; Figure 1, B and E), and DsRed expression from $\mathrm{pXeX}$-DsRed was detected significantly later than either GFP or $\mathrm{BFP}$, at the late gastrula stage $(9 \mathrm{~h}$ of development; Figure 1, C and F). DsRed is well suited for expression

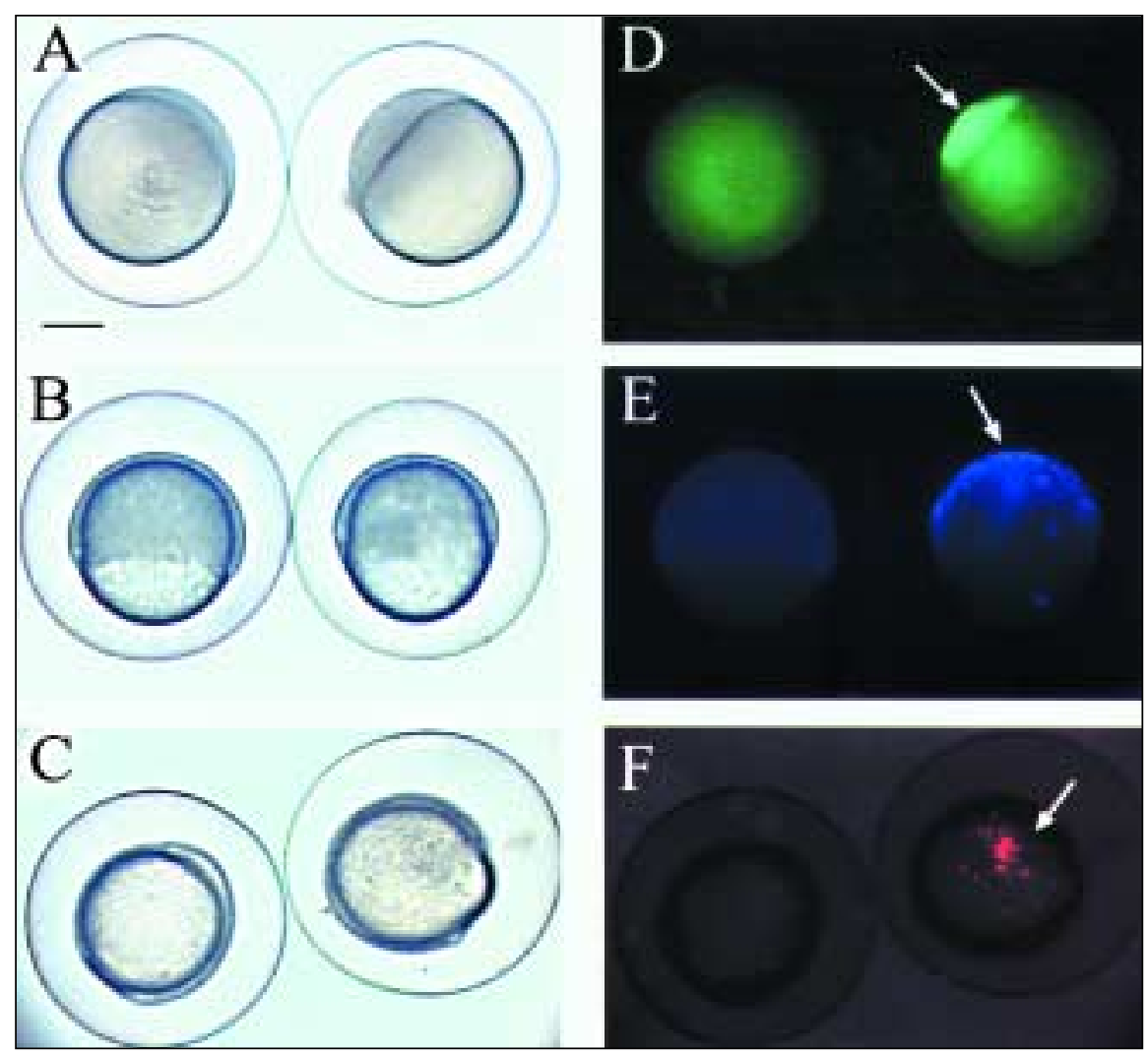

Figure 1. GFP, BFP, and DsRed are first detected at different developmental stages. Injection of pT$\beta$-GM2 resulted in detectable GFP expression at the late sphere stage (A, bright field; D, bp-GFP filter set). Protein from pXeX-BFP was first detected at the shield stage (B, bright field; E, b-GFP filter set), while expression from $\mathrm{pXeX}$-DsRed appeared later at the tail bud stage $(\mathrm{C}$, bright field; F, rhodamine filter set). Scale bar $=250 \mu \mathrm{m}$. 


\section{Short Technical Reports}

analysis because of its particularly high signal-to-noise ratio, especially after 30 $\mathrm{h}$ of development where background fluorescence in the FITC channel becomes apparent (data not shown).

Under standard viewing conditions, GFP and DsRed are exceptionally photostable, while BFP is detectably photolytic (Figure 2). After 15 min of continuous excitation at the appropriate wavelengths, GFP and DsRed showed no reduction in signal at $15 \mathrm{~min}$ (compare Figure 2A with Figure 2D and Figure 2C with Figure 2F) or $45 \mathrm{~min}$ (data not shown). The BFP signal, in contrast, was significantly reduced after 15 min of continuous exposure to the excitation light (Figure 2, B and E).

We compared these fluorescent proteins for sensitivity and specificity of signal for single- and multi-protein applications. Using a standard FITC filter set (lp-GFP), GFP was readily detected
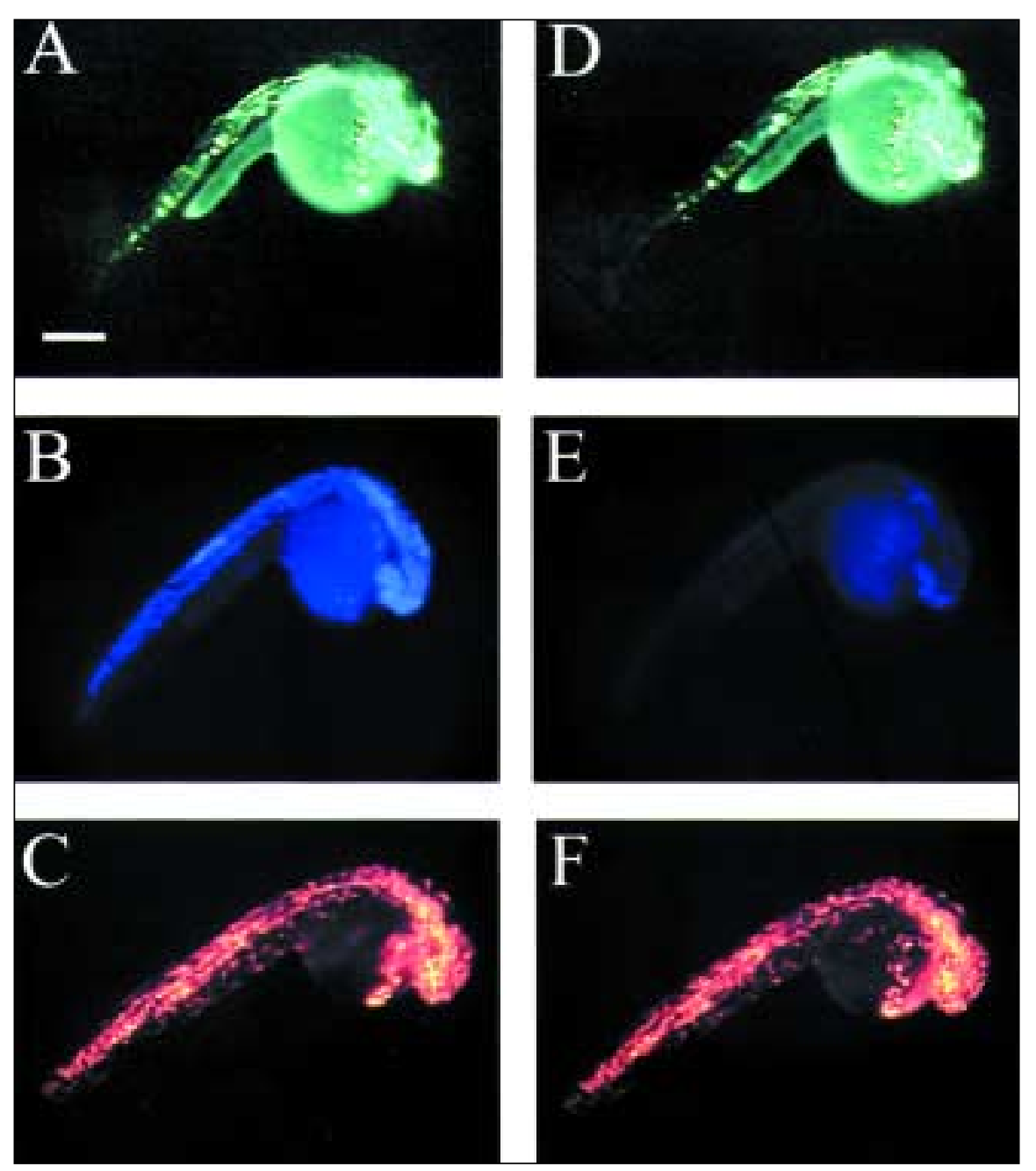

(Figure 3A). However, DsRed was also detected as an orange-pink signal in these embryos (Figure 3C). The emission maximum of DsRed from Discosoma sp. is $583 \mathrm{~nm}(10)$, which is visible in the HQ500LP emission filter. We changed to a bandpass to exclude the DsRed signal (Figure 3F). The excitation wavelength of the bp-GFP filter set spans 450-490 nm, and the wavelength of the lower energy photons emitted by the chromophore of DsRed is too long to be detected by the restricted bandpass filter (500-550 nm). The change to a bandpass filter (HQ525/50m) for GFP detection also increased the signal-to-noise ratio of GFP (compare Figure 3A and Figure 3D) by excluding longer wavelengths above $550 \mathrm{~nm}$, thereby reducing noise. As a result, shorter exposure times were often sufficient for visualizing GFP fluorescence. In Figure 3D, autofluorescence of the

68 BioTechniques fish proper is greatly reduced, and a number of patches of cells can be seen in the trunk that were not visible in lpGFP analyses (Figure 3A).

BFP detection is readily achieved using a filter set designed to maximize excitation while avoiding the emission spectra of GFP or DsRed (Figure 3, E, $\mathrm{H}$, and $\mathrm{K}$ ). However, the detection of BFP is primarily limited by the high level of background fluorescence of the zebrafish embryo. For example, exposure times that clearly outline the wildtype embryo under conditions for the detection of BFP (Figure $3 \mathrm{H}$ ) yield no visible signal under conditions that detect GFP (Figure 3E) or DsRed (Figure $3 \mathrm{~K})$. This high background coupled with photoinstability limits the utility of BFP for some applications.

The excitation and emission spectra of DsRed are excellent for rhodamine (Figure 3L) or TRITC filter sets (10). The excitation and emission wavelengths of the rhodamine filter set we used $(546+/-10 \mathrm{~nm}$ and $570+\mathrm{nm}$, respectively) resulted in invisible uninjected zebrafish, except under long camera exposure times (typically greater than $0.5 \mathrm{~s})$. Compared to injections with pT$\beta-\mathrm{GM} 2$ or $\mathrm{pXeX}$-BFP, embryos injected with pXeX-DsRed exhibited an unusually large number of labeled cells. Distribution also usually appears more even and less discrete when compared with embryos injected with GFP or BFP DNA. We believe this to be due to the unusually high signal-to-noise ratio of DsRed at this stage of development. Indeed, discrete, patchy groups of labeled cells that were often seen in embryos injected with DsRed DNA were obscured by the large number of fluorescing cells that surround them. Figure 4, A and C, are representative of the patchy distribution of GFP expressing cells, Figure 4, E and $\mathrm{F}$, are typical for BFP expressing cells, and Figure 4, C and K, show the more even distribution of DsRed.

Figure 2. GFP and DsRed are photostable, while BFP is photolytic. 24-hpf embryos were illuminated for $15 \mathrm{~min}$ with light from the appropriate excitation filter. The starting timepoint $(0$ min) for GFP, BFP, and DsRed is shown in panels A, B, and C, respectively. The 15-min timepoint is shown in panels $\mathrm{D}, \mathrm{E}$, and $\mathrm{F}$ for GFP, $\mathrm{BFP}$, and DsRed, respectively. Loss of signal is only detected for BFP (compare panels B and E). Scale bar $=250 \mu \mathrm{m}$. 


\section{Short Technical Reports}

We investigated the potential use of the three fluorescent proteins for simultaneous labeling experiments. Figure 4 shows the results of double and triple injections into one-cell embryos. Because of the exclusivity of each protein in its respective filter set (Figure 3 ) and the lack of interference from other proteins being expressed (compare Figure 4A with Figure 4E, Figure 4F with Figure $4 \mathrm{~J}$, and Figure $4 \mathrm{C}$ with Figure $4 \mathrm{~K}$ ), detection protocols did not have to be modified. In embryos injected with multiple constructs, few cells that express only one of the proteins were observed. In those cells that fluoresce in multiple channels, light emitted from one protein does not appear to augment or detract from the signals of the other proteins (Figure 4, D, H, and L).

Figure 4, D, H, and L, show the tail of an embryo injected with all three constructs and the inserts demonstrate that individual cells can express the three proteins simultaneously. These panels also show the high signal-tonoise ratio in the bp-GFP filter set (Figure 4D), the high background fluores- cence in the b-GFP filter set (Figure $4 \mathrm{H}$ ), and the propensity of DsRed to label a larger number of cells (Figure 4L) than GFP or BFP.

\section{DISCUSSION}

Despite its late onset of expression, DsRed will be a valuable fluorescent marker because of the high level of detection from this protein, which is due in part to its low turnover rate. For example, when synthetic mRNA encoding DsRed was injected into eight-cell Xenopus embryos, DsRed activity was detected until week four, while the GFP signal was significantly attenuated by the end of the first week (10). Under mosaic inheritance conditions, the extreme stability of DsRed suggests that cells exhibiting DsRed activity may not necessarily contain DsRed DNA or RNA. This effect may be exacerbated by the ability to detect DsRed at low copy number because of the high signal-to-noise ratio in the rhodamine filter set. In the lp-GFP filter set, GFP seems to have a somewhat lower signal-tonoise ratio. In b-GFP, BFP has the lowest signal-to-noise ratio of the three proteins. Comparing GFP-injected embryos (Figure 4D) and BFP-injected embryos (Figure $4 \mathrm{H}$ ) reveals a lower number of detectable BFP-positive cells. This can also be seen comparing DsRed (Figure 4L) and GFP-injected embryos (Figure 4D): fewer GFP-positive cells are detected compared to the number of DsRed-positive cells. Consequently, for many double-labeled lineage applications, BFP and GFP are the fluorescent proteins of choice.

The photostability of GFP and DsRed make them useful markers in contexts where they will be subjected to frequent or sustained illumination by exciting wavelengths. BFP requires care to minimize its exposure to wavelengths of light that cause its photodegradation. In another photostability assay where we subjected 24-hpf embryos to unfiltered white light from the 100-W halogen source on a Zeiss Axioplan2 microscope, BFP was only mildly photolytic over the course of $1 \mathrm{~h}$ (unpublished re-
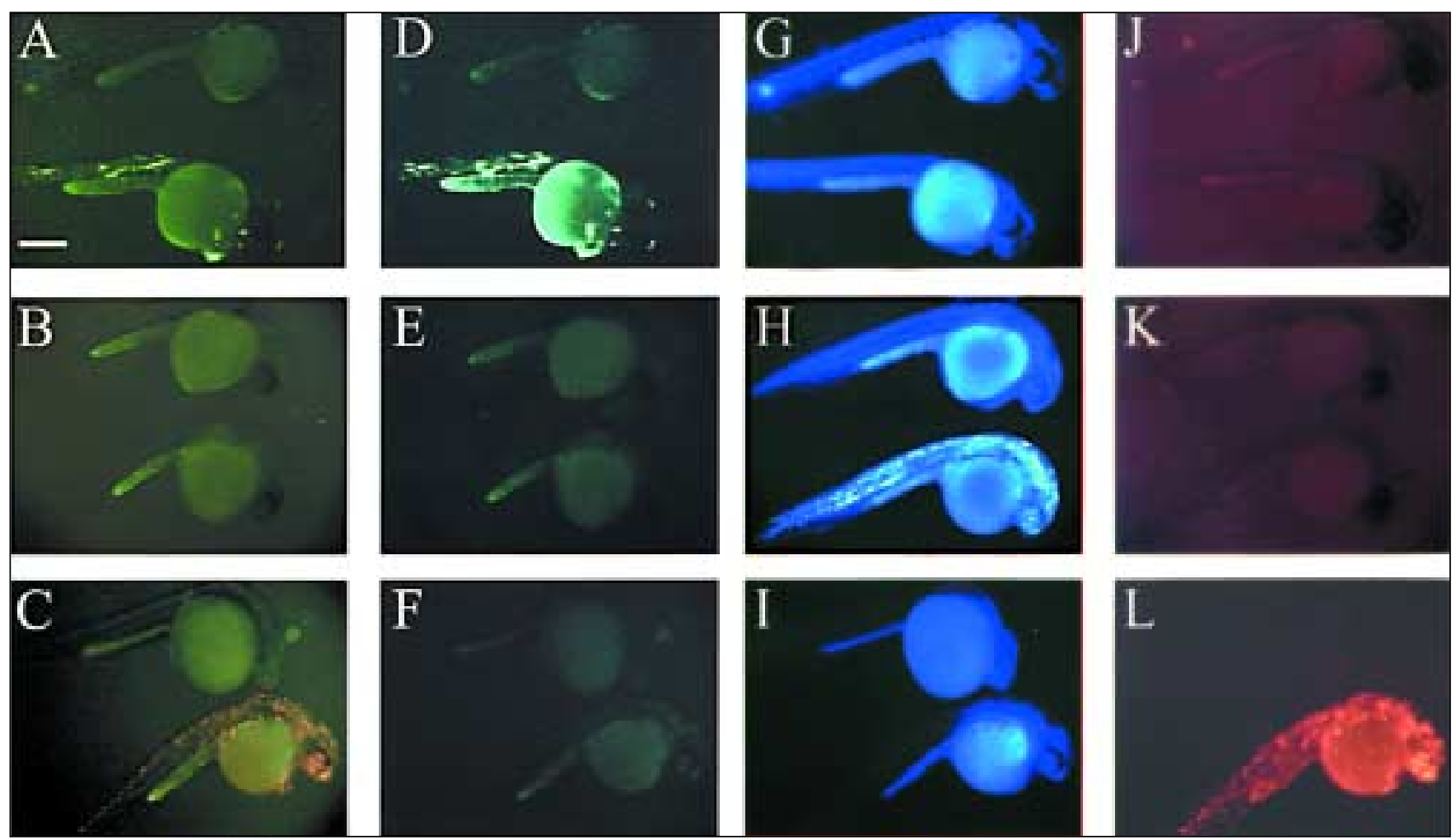

Figure 3. Exclusive detection of GFP, BFP, and DsRed is possible. Three pairs of 24-hpf embryos: uninjected (top), injected (bottom). Embryos were injected at $0 \mathrm{~h}$ with GFP (A, D, G, and J), BFP (B, E, H, and K), or DsRed (C, F, I, and L) and examined under each filter set for overlap detection (A-C, lp-GFP filter set; D-F, bp-GFP filter set; G-I, b-GFP filter set; J-L, rhodamine filter set). DsRed bleeds through the longpass emission filter of the lp-GFP filter set (C). Use of a bandpass emission filter increases GFP signal-to-noise ratio (D) and excludes DsRed (F). Scale bar $=250 \mu \mathrm{m}$. 


\section{Short Technical Reports}
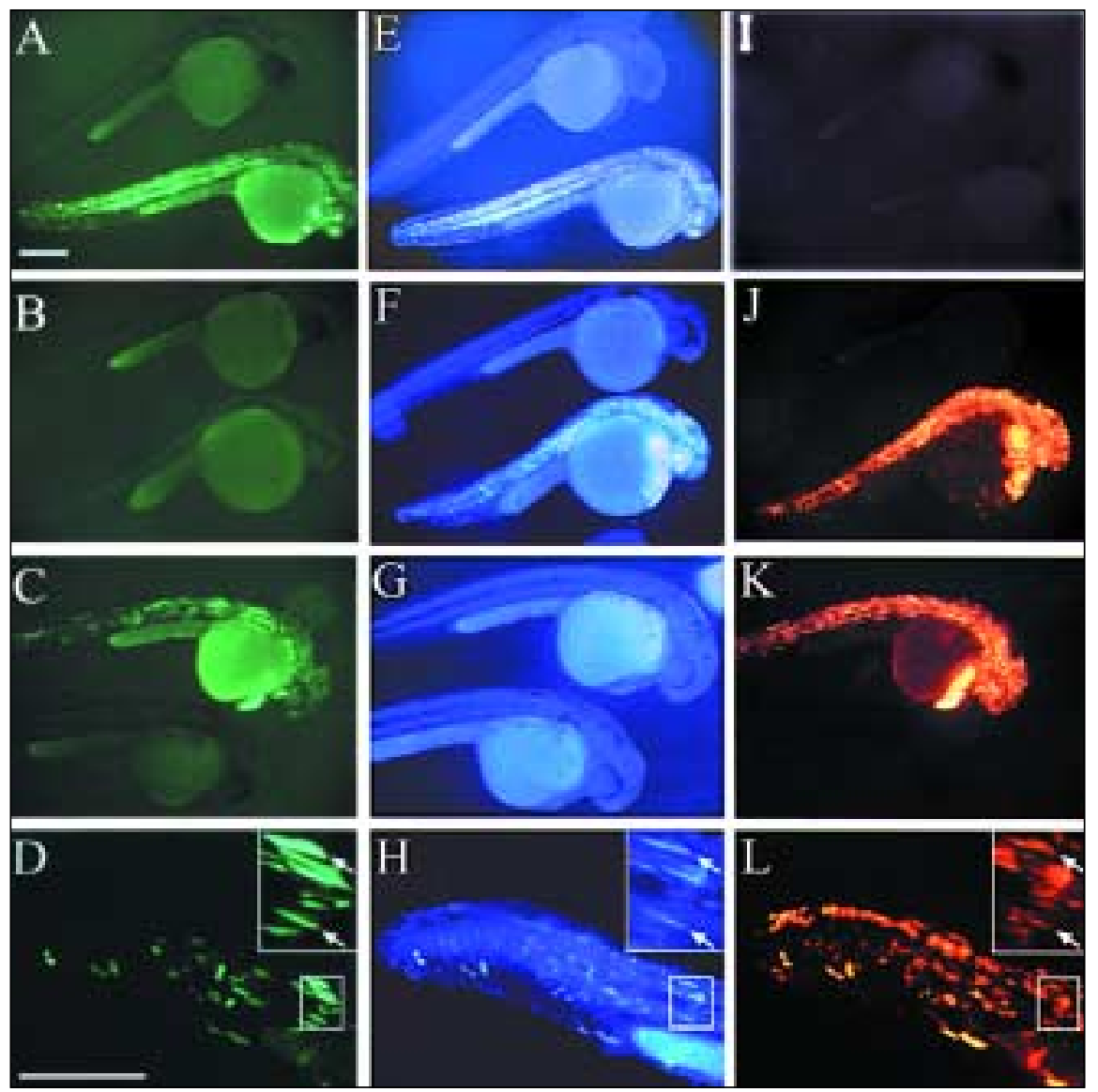

Figure 4. Double and triple label experiments can be documented without false color imaging. Embryos were injected in pairwise combinations of GFP, BFP, and DsRed (A, E, and I, BFP and GFP; B, F, and J, BFP and DsRed; C, G, and K, GFP and DsRed) or co-injected together (D, H, and L). Images of doubly injected fish were taken at a later developmental stage than the triply injected fish. Panels A-D were photographed under the bp-GFP filter set, E-H under the b-GFP filter set, and I-L were taken under the rhodamine filter set. Panels D, H, and L are magnified to show the ability of the fluorescent proteins to express in single cells. Arrows indicate cells in the dermamyotome that simultaneously express all three fluorescent proteins. Images are real-color, enhanced for printing. Scale bars $=250 \mu \mathrm{m}$.

sults). We have modified our protocols when working with BFP to minimize its exposure to ambient light.

Because of the optical clarity of the early zebrafish embryo, fluorescent reporters alleviate the dependence on molecular probing for transgenes by providing a visual indicator that the vector of interest is present. Additionally, fluorescent labeling can provide the same information obtained from in situ hybridization or immunohistochemical staining. These benefits reduce time, reagents and the number of zebrafish used. Creative expression vectors and insightful experiments using multiple fluorescent proteins will help elucidate signaling pathways and regulatory elements and simplify insertional mutagenesis studies.

\section{REFERENCES}

1.Amsterdam, A., S. Lin, and N. Hopkins. 1995. The Aequorea victoria green fluorescent protein can be used as a reporter in live zebrafish embryos. Dev. Biol. 171:123-125.

2.Amsterdam, A., S. Lin, L.G. Moss, and N. Hopkins. 1996. Requirements for green fluorescent protein detection in transgenic zebrafish embryos. Gene 173:99-103.

3.Chalfie, M., Y. Tu, G. Euskirchen, W.W. Ward, and D.C. Prasher. 1994. Green flourescent protein as a marker for gene expression. Science 263:802-805.

4.Essner, J.J., J.G. Laing, E.C. Beyer, R.G. Johnson, and P.B. Hackett. 1996. Expression of zebrafish connexin43.4 in the notochord and tail bud of wild-type and mutant no tail embryos. Dev. Biol. 177:449-462.

5.Heim, R. and R.Y. Tsien. 1996. Engineering green fluorescent protein for improved brightness, longer wavelengths and fluorescence resonance energy transfer. Curr. Biol. 6:178182.
6.Hyatt, T.M. and S.C. Ekker. 1999. Vectors and techniques for ectopic gene expression in zebrafish. Methods Cell. Biol. 59:117-126.

7.Johnson, A.D. and P.A. Krieg. 1994. pXeX, a vector for efficient expression of cloned sequences in Xenopus embryos. Gene 147:223226.

8.Linney, E., N.L. Hardison, B.E. Lonze, S. Lyons, and L. DiNapoli. 1999. Transgene expression in zebrafish: a comparison of retroviral-vector and DNA-injection approaches. Dev. Biol. 213:207-216.

9.Long, Q., A. Meng, H. Wang, J.R. Jessen, M.J. Farrell, and S. Lin. 1997. GATA-1 expression can be recapitulated in living transgenic zebrafish using GFP reporter gene. Development 124:4105-4111.

10.Matz, M.V., A.F. Fradkov, Y.A. Labas, A.P. Savitsky, A.G. Zaraisky, M.L. Markelov, and S.A. Lukyanov. 1999. Fluorescent proteins from nonbioluminescent Anthozoa species. Nat. Biotechnol. 17:969-973.

11.Motoike, T., S. Loughna, E. Perens, B.L. Roman, W. Liao, T.C. Chau, C.D. Richardson, T. Kawate, J. Kuno, B.M. Weinstein, D.Y. Stainier, and T.N. Sato. 2000. Universal GFP reporter for the study of vascular development. Genesis 28:75-81.

12.Stauber, R.H., K. Horie, P. Carney, E.A. Hudson, N.I. Tarasova, G.A. Gaitanaris, and G.N. Pavlakis. 1998. Development and applications of enhanced green fluorescent protein mutants. BioTechniques 24:462-471.

13.Westerfield, M. 1995. The Zebrafish Book. University of Oregon, Eugene, OR.

We thank Paul Millman for multiple discussions regarding filter sets and their use in real-time in vivo imaging. We thank Deanna Mohn and Perry Hackett for the use of pT- $\beta$-GM2 before publication. Many thanks to Dawn Demmon and Jon Larson for help in constructing plasmid $\mathrm{pXeX}$ DsRed. This work was supported by the Arnold and Mabel Beckman Foundation for Transposon Research at the University of Minnesota. Address correspondence to Dr. Stephen C. Ekker, Arnold and Mabel Beckman Center for Transposon Research at the University of Minnesota, Department of Genetics, Cell Biology, and Development, 6-160 Jackson Hall, 321 Church St. SE, Minneapolis, MN 55455, USA. e-mail: ekker001@mail.med.umn.edu

Received 7 July 2000; accepted 12 February 2001.
Kenneth R. Finley,
Ann E. Davidson, and
Stephen C. Ekker
University of Minnesota
Minneapolis, MN, USA 